\title{
Formulation and evaluation of Duloxetine loaded bio-nano suspension for brain specificity via acoustic meatus
}

\author{
N.V.Satheesh Madhav ${ }^{1 *}$, Deepika Raina ${ }^{2}$ \\ ${ }^{1}$ Director, DIT University, Makkawala, P.O Bhagwantpura-248009, Dehradun, Uttrakhand, India \\ ${ }^{2}$ Research scholar, DIT University, Makkawala, P.O Bhagwantpura-248009, Dehradun, Uttrakhand, India
}

Received: October 12, 2016; Accepted: November 10, 2016; Published: January 5 ,2017

*Corresponding author: N.V.Satheesh Madhav, Director, DIT University, Makkawala, P.O Bhagwantpura-248009, Dehradun, Uttrakhand, India, Tel: +91 9760203573; Fax no: 3000309; Email: satheesh_madhav@yahoo.com

\begin{abstract}
The aim of the current research work was to formulate drug loaded nano-suspension using novel bioexcepient isolated from kernels of Prunus amygdalus and to explore the capability of external acoustic meatus as novel acoustic drug delivery system. The biomaterial was extracted by simplified economical process and was further purified by hot dialysis method. The isolated biomaterial was subjected to various physicochemical evaluations along with spectral analysis including UV, IR, Mass, NMR, SEM. The bio-nano suspension formulated with the novel bioexcepient was tested for its functional properties, like retardability, stability and mucoadhesitivity using shear stress method. Different formulations of duloxetine were prepared by using biomaterial as a retardant cum stabilizer and glycerin as nanosizent. The formulations were subjected to various evaluations, including $\mathrm{pH}$, dispersibility, Entrapment efficiency, Particle size, mucoadhesion, skin irritancy, in-vitro release, for over 36 hours Stability. Experimental result reveal that the biopolymer posses promising retardibility cum stability and mucoadhesivity. According to the in-vitro and pharmacodynamic results obtained it can be concluded that significant amount of drug reaches to the brain via external acoustic meatus and so it is feasible to deliver Duloxetine by acoustic meatus.
\end{abstract}

Key words: Acoustic meatus; Duloxetine; Nano-suspension Retardability; Prunus amygdalus

\section{Introduction}

The auricular skin cartilage has a unique histology with a thickness of about $0.8-1.2 \mathrm{~mm}$ in thickness that is securely seized to the perichondrium and also consists of convoluted elastic cartilage lacking blood vessels of 1.0-3.0 mm in thickness. External auricle cartilages are composed of three groups: (1) a ring-like cartilage at the putative tragus, (2) two or three barlike cartilages along the inferoposterior wall of the external acoustic meatus, and (3) a plate-like cartilage in a skin fold for the putative helix of the auricle. The outer epithelium of the tympanic membrane develops from the external acoustic meatus. the outer more superficial surfaces of the auricle are supplied by the great auricular nerve (anterior and posterior inferior portions) and the lesser occipital nerve (posterior superior portion) from the cervical plexus and the auriculotemporal branch of the mandibular nerve $\left[\mathrm{V}_{3}\right]$ (anterior superior portion); the deeper parts of the auricle are supplied by the vagus nerve [X] (the auricular branch) and the facial nerve [VII] (which sends a branch to the auricular branch of the vagus nerve [X]). (4) These unique features serve as a drug delivery platform for effective delivery of API molecules.

The economic cost of depression and its treatment are estimated at $\$ 6$ billion in Canada, US $\$ 83$ billion and $£ 118$ billion. Depression is a chronic, recurring and potentially life-threatening illness that affects up to $20 \%$ of the population across the globe. The etiology of the disease is suboptimal concentrations of the monoamine neurotransmitters serotonin (5-HT) and norepinephrine (NE) in CNS. It is also due to consequence of dysfunctional endocrine, immune and neurotransmitter systems.

Duloxetine is an antidepressant that blocks the reuptake of both serotonin (5-HT) and norepinephrine (NE) by presynaptic transporters and also has low affinity for muscarinic cholinergic, histamine H1, 1 adrenergic as well as serotonin and dopamine receptors (5). Duloxetine exhibits seven to nine-fold greater potency at blocking ligand binding to the serotonin transporter (SERT) than the norepinephrine transporter (NET) and is about 3.5 times more potent at blocking [3H] 5-HT uptake than that of [3H] NE. (6). Duloxetine shows a very different distribution pattern. Very low concentrations in the cerebrospinal fluid is seen may be due to the fact that the drug crosses the bloodcerebrospinalfluid barrier much worsethan otherantidepressants do, suggesting a low ability of duloxetine to enter the brain. Alternatively, low drug concentrations may be interpreted in a sense of a missing residence time in cerebrospinal fluid due to active transport mechanisms out of this environment either back into the bloodstream or into the brain. (7) Aim of the research was to design a suitable formulation loaded with Duloxetine using a novel biopolymer from kernels of Prunus amygdalus as a bio-retardent and stabilizer for delivering nanosized Duloxetine molecule through trans acoustic epithelial route for improved therapeutic efficacy of API.

\section{Material and Methods}

Duloxetine was obtained as a gift sample from INTAS Dehradun. Biomaterial was obtained from the local market; remaining materials used were of analytical grade. 


\section{Isolation of bio-material from the seeds of Prunus amygdalus}

250 grams of Prunus amygdalus kernels were soaked in double distilled water for $24 \mathrm{Hrs}$. The outer cover was removed, and was grinded into a paste. $300 \mathrm{ml}$ water was added to the paste and it was filtered through a muslin cloth. The filtrate was centrifuged at $5000 \mathrm{rpm}$ for 30 minutes to remove the residual matter. The optimized non aqueous solvent propanone in ratio of 1:1 was used for isolation of bio-polymer. Bio-material was separated by centrifugation at $4000 \mathrm{rpm}$ for 10 minutes. The bio-material was dried in vacuum desiccators for $48 \mathrm{hrs}$ and was purified by dialysis membrane [7].

\section{Nano-sizing of Duloxetine}

To $100 \mathrm{mg}$ of duloxetine, $5 \mathrm{ml}$ methanol was mixed and triturated. $5 \mathrm{ml}$ distilled water was added slowly \& sonicated for 5 cycles ( 1 cycle for $3 \mathrm{~min}$.). After each sonication cycle absorbance $\& \% \mathrm{~T}$ were measured. It was then micro centrifuged. Supernatant and residue were collected. Residue was dried \& nanoparticles were recovered.

\section{Drug Excepient study}

The pure drug along with the formulation excipients were subjected to interaction study by U.V Spectroscopy. The study was carried out by dry and wet mixing of the drug and excipient in ratios of 1:1, 1:3, 3:1. Both the mixture was stored at room temperature and at $55^{\circ} \mathrm{C}$ for three days. The dilution was made by the solvent and the sample was scanned at $\lambda$ max using UV spectroscopy.

\section{Permeability}

Drug solution of $1 \mathrm{mg} / \mathrm{ml}$ was prepared and $1 \mathrm{ml}$ drug solution poured in donor compartment. PH7.2 buffer was prepared and was kept in the receptor compartment. The sample was replaced completely every time. Egg membrane was used as a biological membrane as it mimics the action of the ear biological membrane

\section{Formulation of bio nano suspension}

Nanosuspensions were prepared by sonication method using Prunus amygdalus as retardant and with other co-processing agent like glycerin and dextrose as a nanosizent. Weighed amount of drug, dextrose and polymer was triturated together in mortar and pestle and kept on sonicator. Glycerin was added to above mixture in sonication mode. Eight formulations were prepared viz. F1(1:0.5) , F2(1:1), F3((1:2), F4(1:3),F5(1:4), F6(1:5),F7(1:7), F8(1:10).The formulations were subjected to various evaluations parameters

\section{Physico-chemical characterization of the bio-polymer}

The isolated bio-material was check for color, odor, taste, solubility, color changing point, and viscosity. The biopolymer was also tested for the presence of carbohydrates and proteins.

\section{SEM Analysis}

The SEM analysis of the bio-polymer revealed that it has a smooth surface with no rough edges. It shows the smooth, amorphous nature of the bio-polymer. The bio-polymer showed a morphological structure similar to the polymers and hence it confirms the polymeric nature of the bio-polymer (Fig 2).

\section{In-vitro adhesive study using the shear stress method}

The adhesive property of the isolated biomaterial was determined by In-vitro shear stress method. Three different concentration of the biomaterial $(1 \%, 3 \%, 5 \%)$ were placed between two glass plates and subjected to shear stress for assessment of in-vitro adhesive strength in terms of weight required for breaking adhesive bonds between the material and the glass plate after a specified contact time of 5,10,15 and 30 minutes.

\section{Results and discussion}

\section{Isolation of bio-material from the seeds of Prunus amygdalus}

The \% yield for P. amygdalus was found to be $10.2 \pm 2.33 \%$ with a color changing point of $275^{\circ} \mathrm{C} \pm 5^{\circ} \mathrm{C}$. The bio-materials were purified and no presence of chlorides, sulphates and starch was observed

\section{Nano-sizing of Duloxetine}

When a sample is subjected for measurement of $\% \mathrm{~T}$ at different wavelengths the percentage of transmittance reflects the percentage of the particles which are present in the mixture below $400 \mathrm{~nm}$. Whereas the \% blockade indicates the \% particle which are above $400 \mathrm{~nm}$ and the data was correlated with the SEM analysis.

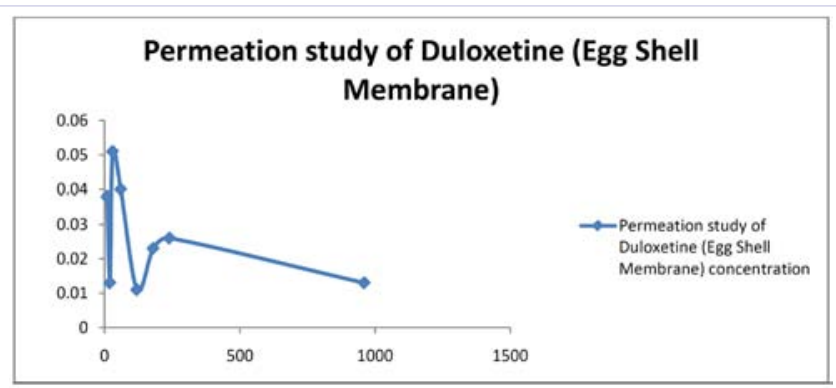

Figure 1:

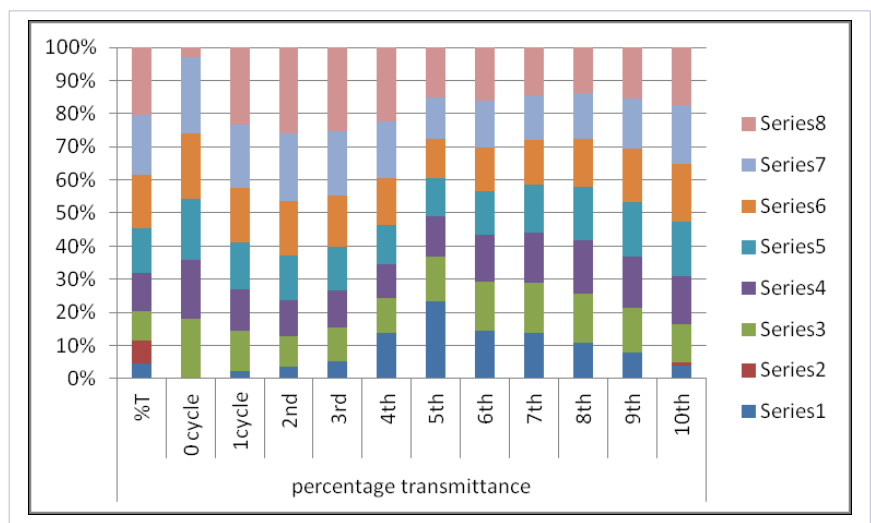

Figure 2: Nanosizing 


\section{Drug Excepient study}

The drug interaction study revealed that there was no interaction between the drug and the excipients including the bio-polymers. This was proved by the result of the thin layer chromatography in which no change was seen in the RF value in the TLC method. Also there was no change in the $\lambda$ max by UV method. Value which was observed to be $289 \mathrm{~nm}$ prior to the test and after the test it was $289 \mathrm{~nm}$ hence confirming that there was no interaction between the drug and excipients. No observable signs of drug interaction were seen. It was concluded that none of the excipients had a detrimental effect on the drug and could be safely used for the formulation of the suspension.

\section{Permeability}

Egg membrane was used as a biological membrane as it mimics the action of the ear biological membrane. A permeation graph was plotted between concentration vs time, depicting the amount of drug permeated.

\section{Physico-chemical characterization of the bio-polymer}

The isolated bio-material was white in colour, odourless, characteristic taste, partially soluble in water, colour changing point of $270-275^{\circ} \mathrm{C}$. It had a viscosity of $1.54 \mathrm{cps}$, carbohydrates were absent while proteins were present (Table 1 ).

The IR spectra revealed the presence of tertiary $(1078.13 \mathrm{~cm}$ 1), secondary alcohols, (1151.13 cm-1) aromatic rings(1598.88 $\mathrm{cm}-1)$ and the presence of alkanes, alkenes(2925.81 cm-1) and nitro compounds along with ketones(1678.5 cm-1)(fig. no I). These groups like the ketonic groups, nitro groups indicate the mucoadhesive activity of the bio-polymer as these groups are observed in the mucoadhesive polymers like HPMC, polycarbophil. The mass spectra shows the large molecular weight structure showing the polymer nature and the presence of proteins. It showed a parent peak at $701.7 \mathrm{~m} / \mathrm{z}$ value (Fig 3). The NMR spectra revealed Doublets at 1.2-1.8 and quartet at 4.03 reveals the presence of methyl groups (Fig 4). The isolated biomaterial was further evaluated for its adhesivity by using shear stress method.

\section{Characterization of drug loaded nano suspension (Table 2)}

pH studies: value of $\mathrm{pH}$ was noted from digital $\mathrm{pH}$ meter. The method was performed in triplicate \& mean value of $\mathrm{pH}$ was calculated and was found between 7.2-7.8 (Table 3).

Dispersibility: Evaluation of dispersibility of nanoparticles was done by manual hand shaking method. $10 \mathrm{mg}$ of accurately weighed nanoparticles were taken in test tube \& dispersed in 10 $\mathrm{ml}$ of double distilled water. After dispersion of the nanoparticles time taken for settling of particles to the bottom of the test tube was noticed \& redispersion of nanoparticles on shaking of test tube was noticed. Visual observation was done to investigate formation of any aggregates or precipitates after shaking.

Entrapment efficacy: Entrapment efficacy was calculated to find out the amount of entrapped drug inside the nanoparticles.

\begin{tabular}{|c|c|c|}
\hline \multicolumn{3}{|c|}{ Table 1: Characterization of biopolymer } \\
\hline 1 & Color & White \\
\hline 2 & Odor & Odorless \\
\hline 3 & Taste & Characteristic \\
\hline 4 & Solubility & Partially soluble in water \\
\hline 5 & Melting point & $270-275$ \\
\hline 6 & Proteins & Present \\
\hline 7 & Carbohydrates & Absent \\
\hline
\end{tabular}

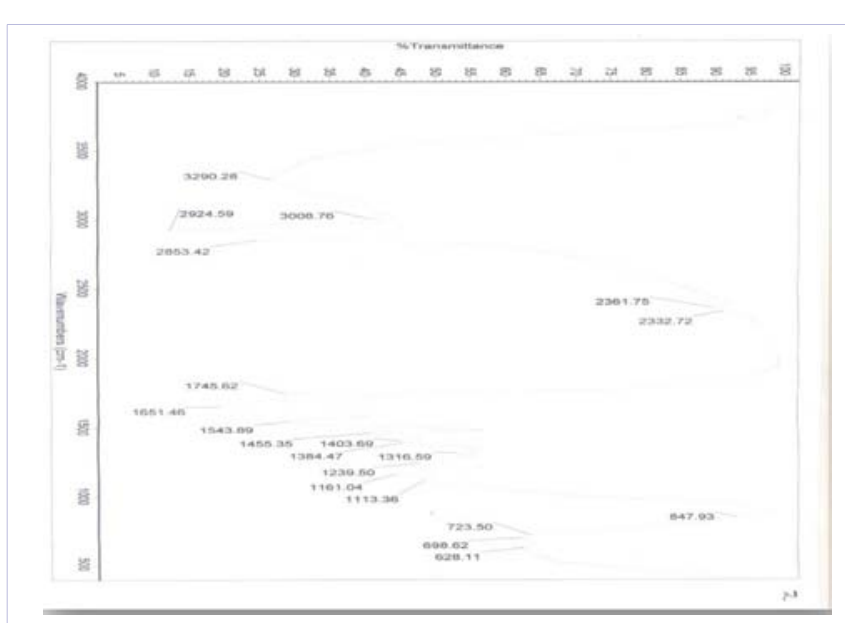

Figure 3: IR Spectra of Prunus amygdalus biopolymer

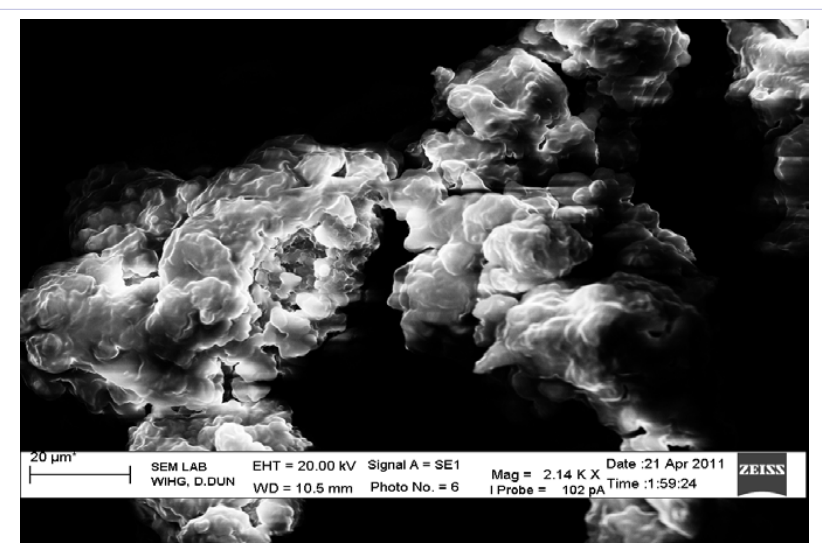

Figure 4: SEM of Prunus amygdalus

It was calculated by accurately weighing $5 \mathrm{mg}$ of formulated nanoparticles \& dissolving them in $5 \mathrm{ml}$ of methanol. The solution was sonicated for 10 mins \& kept for $24 \mathrm{hrs}$ as such. After $24 \mathrm{hrs}$ each solution was diluted up to $10 \mu \mathrm{g} / \mathrm{ml} \&$ was analyzed under UV at $289 \mathrm{~nm}$ against the blank methanol solution \& drug content was calculated. Entrapment efficacy was calculated by following formula-

Entrapment efficacy- amount of drug in nanoparticles/drug added in nanoparticles* 100

Preliminary method to screen the nano particle size range by UV method: Transmittance of the nanosuspensions 
was studied as preliminary study for the particle size analysis. It gave an idea regarding the particle size of the nanosuspensions formulation. Transmittance is based on the concept of Tindal effect which specify that when light of specified wavelength passes through the media containing particles less than or greater than specified particle range, the \% blockage represent particle beyond size range at particular range whereas \% Transmittance is considered that the particles lies above the size range at particular range. Transmittance of the formulation was studied by UV spectroscopy between 400-600 nm ranges keeping plain double distilled water as the blank. The reading showed the number of particles that allow the UV light to pass through it \& rest of the particles showed the range of particles that blocked the light thus providing an idea of the range of particles in the nanosuspension.

Particle size (Size Distribution by Intensity): Preliminary study for particle size study by $\%$ transmittance was followed by Particle size range \& size distribution study of the nanosuspension. Nanoparticle size was studied \& average diameter range \& intensity of the particles in particular size range was studied.

Skin irritancy test for nanosuspension: Irritancy test was carried out to determine possible localized reaction of the selected formulation to the ear skin. A single dose of $1 \mathrm{ml}$ of the selected formulations was administered to the right ear of male albino rat and the left side was considered as control. The development of erythema was monitored daily for 6 days in both the ears \& presence of any type of skin reaction or allergic reaction was noticed.

In-Vitro studies: The in-vitro release pattern of FA1-FA8 were studied by dynamic method and a graph is plotted between $\%$ drug release and time, $\mathrm{r}^{2}$ value $\mathrm{t} 50$ and $\mathrm{t} 80$ were calculated from the graph, which showed drug release ranging from 8589\%.(Fig 5 \& 6)

Stability Studies: Stability studies were performed according to ICH guidelines. The formulations were stored in hot air oven at $37 \pm 2^{\circ}, 45 \pm 2^{\circ}, 4 \pm 2^{\circ}$ and $60 \pm 2^{\circ}$ for a period of 3 months. The samples were analyzed for drug content every two weeks by UVVisible Spectrophotometer at $289 \mathrm{~nm}$. Stability study was also

Table 2: Formulation of Duloxetine bio-nanoparticles loaded with Prunus amygdalus biopolymer.

\begin{tabular}{|l|c|c|c|c|c|c|c|c|}
\hline & FA1 & FA2 & FA3 & FA4 & FA5 & FA6 & FA7 & FA8 \\
$\mathbf{( 1 : 0 5 )}$ & $\mathbf{( 1 : 1 )}$ & $\mathbf{( 1 : 2 )}$ & $\mathbf{( 1 : 3 )}$ & $\mathbf{( 1 : 4 )}$ & $\mathbf{1 : 5})$ & $\begin{array}{l}\mathbf{( 1 : 7 )} \\
\mathbf{( 1 : 1 0 )}\end{array}$ \\
\hline $\begin{array}{l}\text { Drug:polymer } \\
\text { ratio }\end{array}$ & $1: 0.5$ & $1: 1$ & $1: 2$ & $1: 3$ & $1: 4$ & $1: 5$ & $1: 7$ & $1: 10$ \\
\hline Duloxetine (mg) & 10 & 10 & 10 & 10 & 10 & 10 & 10 & 10 \\
\hline $\begin{array}{l}\text { Prunus amygdalus } \\
\text { Bio-polymer } \\
\text { (mg) }\end{array}$ & 0.5 & 10 & 20 & 30 & 40 & 50 & 70 & 100 \\
\hline \begin{tabular}{l} 
Glycerin $\mu \mathrm{l}$ \\
\hline Dextrose (mg)
\end{tabular} & 100 & 100 & 100 & 100 & 100 & 100 & 100 & 100 \\
\hline $\begin{array}{l}\text { Distilled } \\
\text { water(ml) }\end{array}$ & 30 & 30 & 30 & 30 & 30 & 30 & 30 & 30 \\
\hline
\end{tabular}

\begin{tabular}{|c|c|}
\hline \multicolumn{2}{|c|}{ Table 3: $\mathrm{pH}$ studies } \\
\hline FA1 & 7.3 \\
\hline FA2 & 7.2 \\
\hline FA3 & 7.5 \\
\hline FA4 & 7.3 \\
\hline FA5 & 7.4 \\
\hline FA6 & 7.4 \\
\hline FA7 & 7.5 \\
\hline FA8 & 7.2 \\
\hline
\end{tabular}

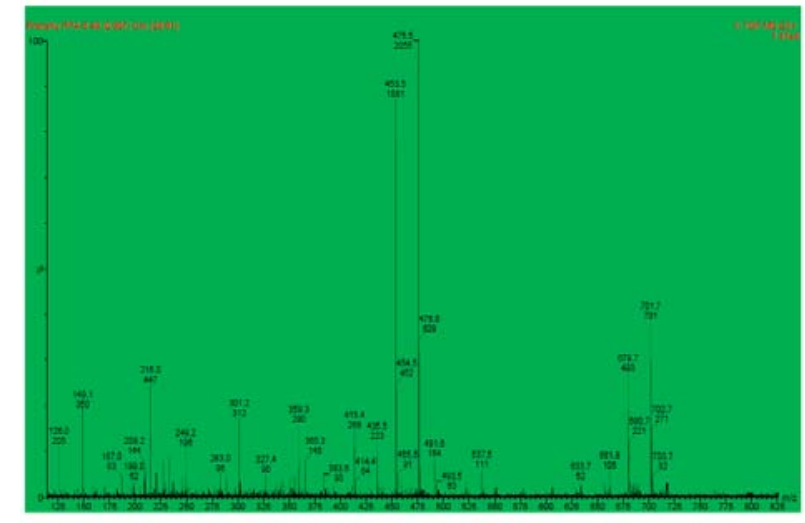

Figure 5: Mass of Prunus amygdalus

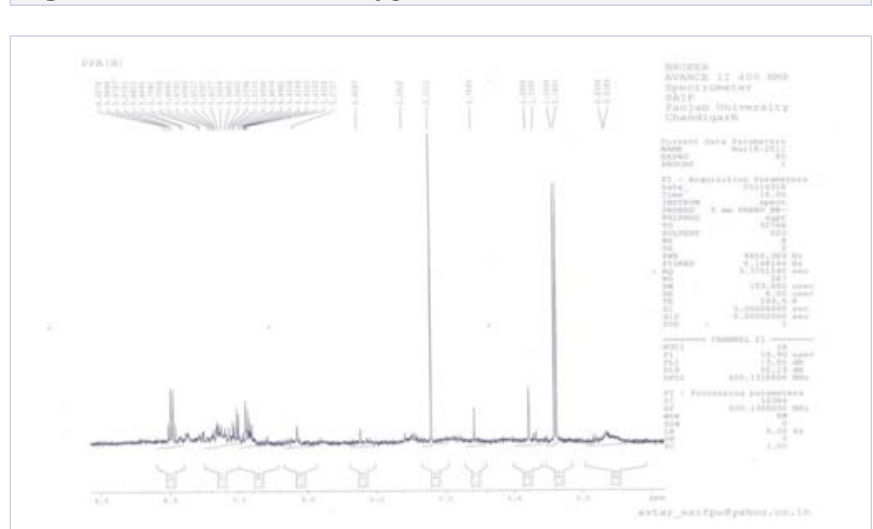

Figure 6: NMR of Prunus amygdalus

carried out by measuring the change in $\mathrm{pH}$ of nano-suspension weekly in terms of change in color, odor, taste, its entrapment efficiency, and In-Vitro drug released.

Duloxetine is an acid labile drug, degrades at acidic $\mathrm{pH}$ of stomach. Duloxetine shows comparably very low concentrations in cerebrospinal fluid, due to the fact that the drug crosses the blood-cerebrospinal fluid barrier much worse than other antidepressants do, suggesting a low ability of duloxetine to enter the brain. Different formulations of duloxetine were prepared out of which FA1 formulation was found to be the best formulation showing a $\mathrm{r}^{2}$ value of 0.9905 T80: 22 hrs and best fit model was found to be higuchi matrix, and mechanism of transport was anomalous transport. In-vitro release pattern over an extended 

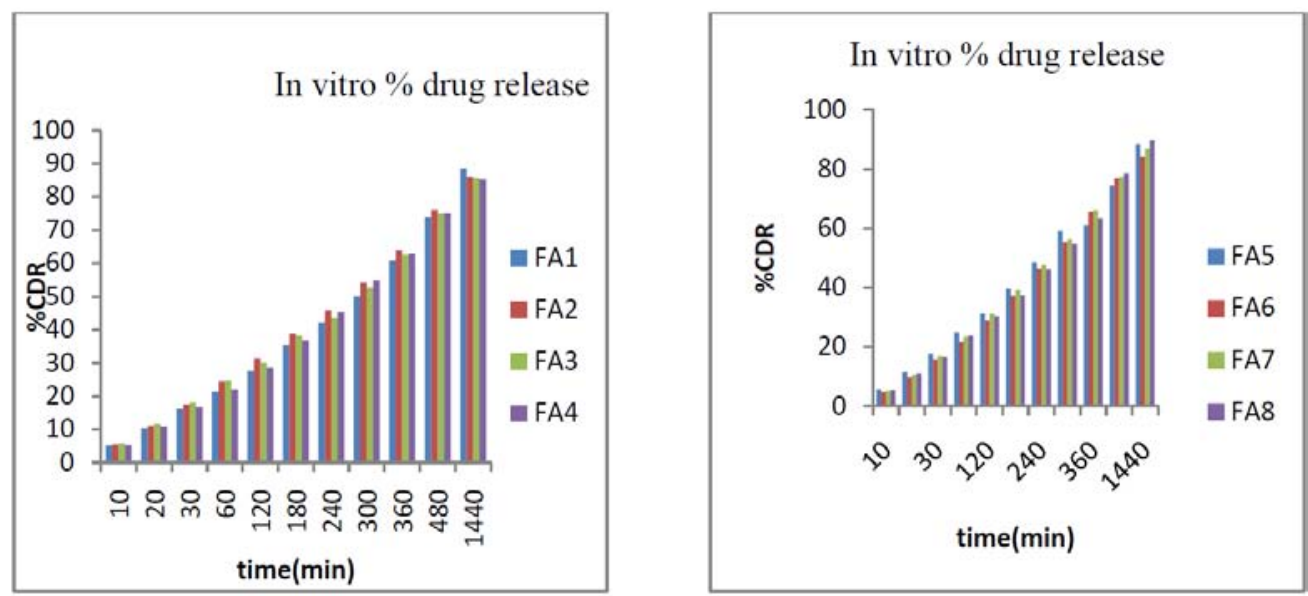

Figure 7,8: IN-Vitro \% drug release

period of time shows that significant amount of drug reaches to the brain.

As there are no pharmaceuticals designed specifically for brain targeting to treat the depression via ear. We have designed a dosage form to combat the disease and increase patient compliance thereby minimizing the incidences of dose missing which are relatively quite high due to busy schedule and long term therapy course thus prevents he precipitation of the disease from chronic stage. The long term therapy and multiple dosing is the main reason for the discomfort of the patient. All above mentioned problem can be overcome by the instilling of Duloxetine loaded nano-suspension in to the ear which Is targeted directly to the brain via inter and intra neural pathway. Proposed mechanism based on the research out coming, for drug targeting to brain from ear may achieve via neural pathway which is located ear and brain through vestibular ganglion to verve VIII and from nerve VII to left cochlear neuron which is located in brain. This route can be used for drug targeting to the brain. Different drugs were given to the rats and reduced activity of rat indicates that drug was entered in to the brain of rat through ear via neural pathway [8].

\section{Acknowledgment}

We thank Prof. K.K Raina and Prof. (Dr.) N.V.Satheesh Madhav for their immense support and assistance that greatly improved the manuscript.

\section{References}

1. Weerda H. Surgery of the auricle: tumors, trauma defects, abnormalities. New York: Thieme. 2007.

2. Yasutoyo Ikari, Katori Y, Ohtsuka A, Rodríguez-Vázquez JF, Abe $\mathrm{H}$, Kawase $\mathrm{T}$, et al. Fetal development and variations in the cartilages surrounding the human external acoustic meatus. Annals of Anatomy. 2013;195(2):128-136. doi: 10.1016/j.aanat.2012.07.009.

3. Mallo M, Schrewe H, Martin JF, Olson EN, Ohnemus S. Assembling a functional tympanic membrane: signals from the external acoustic meatus coordinate development of the malleal manubrium. Development. 2000;127(19):4127-4136.

4. Richard Drake, A Wayne Vogl, Adam Mitchell. Gray's Anatomy for Students. 3rd Edition; 1-2 chapter. Churchil Livingstone. 2015.

5. Karpa, Anttila and Leinonen, et al. An XML based ontology exchange language. 2002;3:1217-1221.

6. Georgianna G. Gould, Javors MA, Frazer A. Effect of chronic administration of duloxetine on serotonin and norepinephrine transporter binding sites in rat brain. Biol psychiatry. 2007;61(2):210215

7. Michael Paulzen, Gründer G, Veselinovic T, Wolf B, Hiemke C, Lammertz SE. Duloxetine enters the brain - But why is it not found in the cerebrospinal fluid. Journal of Affective Disorders. 2016:189:159163. doi: 10.1016/j.jad.2015.08.073.

8. Pallavi Yerramsetty, J Vijaya Ratna, Venkata Ramana Reddy, Praveen Kumar. Formulation, Development and Evaluation of delayed release capsules of Duloxetine Hydrochloride made of different Enteric Polymers. Int J Drug Dev \& Res. 2012,4(1): 117-129. 